\title{
Dynamics of a Fractional Order HIV Infection Model with Specific Functional Response and Cure Rate
}

\author{
Adnane Boukhouima, ${ }^{1}$ Khalid Hattaf, ${ }^{1,2}$ and Noura Yousfi ${ }^{1}$ \\ ${ }^{1}$ Laboratory of Analysis, Modeling and Simulation (LAMS), Faculty of Sciences Ben M'sik, Hassan II University, \\ P.O. Box 7955, Sidi Othmane, Casablanca, Morocco \\ ${ }^{2}$ Centre Régional des Métiers de l'Education et de la Formation (CRMEF), 20340 Derb Ghallef, Casablanca, Morocco \\ Correspondence should be addressed to Khalid Hattaf; k.hattaf@yahoo.fr
}

Received 11 June 2017; Accepted 31 July 2017; Published 27 August 2017

Academic Editor: Yuji Liu

Copyright (C) 2017 Adnane Boukhouima et al. This is an open access article distributed under the Creative Commons Attribution License, which permits unrestricted use, distribution, and reproduction in any medium, provided the original work is properly cited.

We propose a fractional order model in this paper to describe the dynamics of human immunodeficiency virus (HIV) infection. In the model, the infection transmission process is modeled by a specific functional response. First, we show that the model is mathematically and biologically well posed. Second, the local and global stabilities of the equilibria are investigated. Finally, some numerical simulations are presented in order to illustrate our theoretical results.

\section{Introduction}

Fractional order differential equations (FDEs) are a generalization of ordinary differential equations (ODEs) and they have many applications in various fields such as mechanics, image processing, viscoelasticity, bioengineering, finance, psychology, and control theory [1-7]. In addition, it has been deduced that the membranes of cells of biological organisms have fractional order electrical conductance [8].

Modeling by FDEs has more advantages to describe the dynamics of phenomena with memory which exists in most biological systems, because fractional order derivatives depend not only on local conditions but also on the past. More precisely, calculating the time-fractional derivative of a function $f(t)$ at some time $t=t_{1}$ requires all the previous history, that is, all $f(t)$ from $t=0$ to $t=t_{1}$. In addition, the region of stability of FDEs is larger than that of ODEs. Moreover, some previous study compared between the results of the fractional order model, the results of the integer model, and the measured real data obtained from 10 patients during primary HIV infection [9]. This study proved that the results of the fractional order model give predictions to the plasma virus load of the patients better than those of the integer model.
From the above biological and mathematical reasons, we propose a fractional order model to describe the dynamics of HIV infection that is given by

$$
\begin{aligned}
D^{\alpha} T(t) & =\lambda-d T-\frac{\beta T V}{1+\alpha_{1} T+\alpha_{2} V+\alpha_{3} T V}+\rho I, \\
D^{\alpha} I(t) & =\frac{\beta T V}{1+\alpha_{1} T+\alpha_{2} V+\alpha_{3} T V}-(a+\rho) I, \\
D^{\alpha} V(t) & =k I-\mu V,
\end{aligned}
$$

where $T(t), I(t)$, and $V(t)$ represent the concentrations of uninfected $\mathrm{CD} 4^{+} \mathrm{T}$-cells, infected cells, and free virus particles at time $t$, respectively. Uninfected cells are assumed to be produced at a constant rate $\lambda$, die at the rate $d T$, and become infected by a virus at the rate $\beta T V /\left(1+\alpha_{1} T+\right.$ $\left.\alpha_{2} V+\alpha_{3} T V\right)$, where $\alpha_{1}, \alpha_{2}, \alpha_{3} \geq 0$ are the saturation factors measuring the psychological or inhibitory effect. Infected cells die at the rate $a I$ and return to the uninfected state by loss of all covalently closed circular DNA (cccDNA) from their nucleus at the rate $\rho I$. Free virus particles are produced from infected cells at the rate $k I$ and cleared at the rate $\mu V$.

The fractional order derivative used in system (1) is in the sense of Caputo. We use this Caputo fractional derivative for 
two reasons: the first reason is that the fractional derivative of a constant is zero and the second reason is that the initial value problems depend on the integer order derivative only. In addition, we choose $0<\alpha \leq 1$ in order to have the same initial conditions as ODE systems.

On the other hand, system (1) generalizes many special cases existing in the literature. For example, when $\alpha_{1}=\alpha_{2}=$ $\alpha_{3}=0$, we get the model of Arafa et al. [10]. Further, we obtain the model of Liu et al. [11] when $\alpha_{3}=0$. It is very important to note that when $\alpha=1$, system (1) becomes a model with an ordinary derivative which is the generalization of the ODE models presented in [12-15].

The rest of the paper is organized as follows. In the next section, we give some preliminary results. In Section 3, equilibria and their local stability are investigated. In Section 4, the global stability of the two equilibria is established. Numerical simulations of our theoretical results are presented in Section 5. Finally, the paper ends with conclusion in Section 6

\section{Preliminary Results}

We first recall the definitions of the fractional order integral, Caputo fractional derivative, and Mittag-Leffler function that are given in [16].

Definition 1. The fractional integral of order $\alpha>0$ of a function $f: \mathbb{R}_{+} \rightarrow \mathbb{R}$ is defined as follows:

$$
I^{\alpha} f(t)=\frac{1}{\Gamma(\alpha)} \int_{0}^{t}(t-x)^{\alpha-1} f(x) d x,
$$

where $\Gamma(\cdot)$ is the Gamma function.

Definition 2. The Caputo fractional derivative of order $\alpha>0$ of a continuous function $f: \mathbb{R}_{+} \rightarrow \mathbb{R}$ is given by

$$
D^{\alpha} f(t)=I^{n-\alpha} D^{n} f(t),
$$

where $D=d / d t$ and $n-1<\alpha \leq n, n \in \mathbb{N}$.

In particular, when $0<\alpha \leq 1$, we have

$$
D^{\alpha} f(t)=\frac{1}{\Gamma(1-\alpha)} \int_{0}^{t} \frac{f^{\prime}(x)}{(t-x)^{\alpha}} d x
$$

Definition 3. Let $\alpha>0$. The function $E_{\alpha}$, defined by

$$
E_{\alpha}(z)=\sum_{j=0}^{\infty} \frac{z^{j}}{\Gamma(\alpha j+1)}
$$

is called the Mittag-Leffler function of parameter $\alpha$.

Let $f: \mathbb{R}^{n} \rightarrow \mathbb{R}^{n}$ with $n \geq 1$. Consider the fractional order system

$$
\begin{aligned}
D^{\alpha} x(t) & =f(x), \\
x\left(t_{0}\right) & =x_{0},
\end{aligned}
$$

with $0<\alpha \leq 1, t_{0} \in \mathbb{R}$, and $x_{0} \in \mathbb{R}^{n}$. For the global existence of solution of system (6), we need the following lemma.
Lemma 4. Assume that $f$ satisfies the following conditions:

(i) $f(x)$ and $(\partial f / \partial x)(x)$ are continuous for all $x \in \mathbb{R}^{n}$.

(ii) $\|f(x)\| \leq \omega+\lambda\|x\|$ for all $x \in \mathbb{R}^{n}$, where $\omega$ and $\lambda$ are two positive constants.

Then, system (6) has a unique solution on $\left[t_{0},+\infty\right)$.

The proof of this lemma follows immediately from [17]. For biological reasons, we assume that the initial conditions of system (1) satisfy

$$
\begin{gathered}
T(0)=\phi_{1}(0) \geq 0, \\
I(0)=\phi_{2}(0) \geq 0, \\
V(0)=\phi_{3}(0) \geq 0 .
\end{gathered}
$$

In order to establish the nonnegativity of solutions with initial conditions (7), we need also the following lemmas.

Lemma 5 (see [18]). Suppose that $g(t) \in C[a, b]$ and $D^{\alpha} g(t) \in$ $C[a, b]$ for $0<\alpha \leq 1$; then, one has

$$
\begin{aligned}
g(t)=g(a)+\frac{1}{\Gamma(\alpha)} D^{\alpha} g(\xi)(t-a)^{\alpha} & \\
& a<\xi<t, \forall t \in(a, b] .
\end{aligned}
$$

Lemma 6 (see [18]). Suppose that $g(t) \in C[a, b]$ and $D^{\alpha} g(t) \in C[a, b]$ for $0<\alpha \leq 1$. If $D^{\alpha} g(t) \geq 0 \forall t \in[a, b]$, then $g(t)$ is nondecreasing for each $t \in[a, b]$. If $D^{\alpha} g(t) \leq 0 \forall t \in$ $[a, b]$, then $g(t)$ is nonincreasing for each $t \in[a, b]$.

Theorem 7. For any initial conditions satisfying (7), system (1) has a unique solution on $[0,+\infty)$. Moreover, this solution remains nonnegative and bounded for all $t \geq 0$. In addition, one has

(i) $N(t) \leq N(0)+\lambda / \delta$,

(ii) $V(t) \leq V(0)+(k / \mu)\|I\|_{\infty}$,

where $N(t)=T(t)+I(t)$ and $\delta=\min \{a, d\}$.

Proof. It is easy to see that the vector function of system (1) satisfies the first condition of Lemma 4. It remains to prove the second condition. Let

$$
X(t)=\left(\begin{array}{c}
T(t) \\
I(t) \\
V(t)
\end{array}\right),
$$

$$
\zeta=\left(\begin{array}{l}
\lambda \\
0 \\
0
\end{array}\right) .
$$

To this end, we discuss four cases:

(i) If $\alpha_{1} \neq 0$, then system (1) can be written as follows:

$$
D^{\alpha} X(t)=\zeta+A_{1} X+\frac{\alpha_{1} T}{1+\alpha_{1} T+\alpha_{2} V+\alpha_{3} T V} A_{2} X,
$$


where

$$
\begin{aligned}
A_{1} & =\left(\begin{array}{ccc}
-d & \rho & 0 \\
0 & -(a+\rho) & 0 \\
0 & k & -\mu
\end{array}\right), \\
A_{2} & =\left(\begin{array}{ccc}
0 & 0 & -\frac{\beta}{\alpha_{1}} \\
0 & 0 & \frac{\beta}{\alpha_{1}} \\
0 & 0 & 0
\end{array}\right)
\end{aligned}
$$

Moreover, we have

$$
\left\|D^{\alpha} X(t)\right\| \leq\|\zeta\|+\left(\left\|A_{1}\right\|+\left\|A_{2}\right\|\right)\|X\| .
$$

(ii) If $\alpha_{2} \neq 0$, we have

$D^{\alpha} X(t)=\zeta+A_{1} X+\frac{\alpha_{2} T}{1+\alpha_{1} T+\alpha_{2} V+\alpha_{3} T V} A_{3} X$,

where

$$
A_{3}=\left(\begin{array}{ccc}
0 & 0 & -\frac{\beta}{\alpha_{2}} \\
0 & 0 & \frac{\beta}{\alpha_{2}} \\
0 & 0 & 0
\end{array}\right) .
$$

Then,

$$
\left\|D^{\alpha} X(t)\right\| \leq\|\zeta\|+\left(\left\|A_{1}\right\|+\left\|A_{3}\right\|\right)\|X\| .
$$

(iii) If $\alpha_{3} \neq 0$, we have

$$
D^{\alpha} X(t)=\zeta+A_{1} X+\frac{\alpha_{3} T V}{1+\alpha_{1} T+\alpha_{2} V+\alpha_{3} T V} A_{4},
$$

where

$$
A_{4}=\left(\begin{array}{c}
-\frac{\beta}{\alpha_{3}} \\
\frac{\beta}{\alpha_{3}} \\
0
\end{array}\right)
$$

Then,

$$
\left\|D^{\alpha} X(t)\right\| \leq\left(\|\zeta\|+\left\|A_{4}\right\|\right)+\left\|A_{1}\right\|\|X\| .
$$

(iv) If $\alpha_{1}=\alpha_{2}=\alpha_{3}=0$, we have

$$
D^{\alpha} X(t)=\zeta+A_{1} X+V A_{5} X
$$

where

$$
A_{5}=\left(\begin{array}{ccc}
-\beta & 0 & 0 \\
\beta & 0 & 0 \\
0 & 0 & 0
\end{array}\right) \text {. }
$$

Then,

$$
\left\|D^{\alpha} X(t)\right\| \leq\|\zeta\|+\left(\|V\|\left\|A_{5}\right\|+\left\|A_{1}\right\|\right)\|X\| .
$$

Thus, the second condition of Lemma 4 is satisfied. Then, system (1) has a unique solution on $[0,+\infty)$. Next, we show that this solution is nonnegative. From (1), we have

$$
\begin{aligned}
\left.D^{\alpha} T(t)\right|_{T=0} & =\lambda+\rho I \geq 0, \\
\left.D^{\alpha} I(t)\right|_{I=0} & =\frac{\beta T V}{1+\alpha_{1} T+\alpha_{2} V+\alpha_{3} T V} \geq 0, \\
\left.D^{\alpha} V(t)\right|_{V=0} & =k I \geq 0 .
\end{aligned}
$$

According to Lemmas 5 and 6, we deduce that the solution of (1) is nonnegative.

Finally, we prove that the solution is bounded. By adding the first two equations of system (1), we get

$$
D^{\alpha} N(t) \leq \lambda-\delta N(t) \text {. }
$$

Hence,

$$
N(t) \leq N(0) E_{\alpha}\left(-\delta t^{\alpha}\right)+\frac{\lambda}{\delta}\left[1-E_{\alpha}\left(-\delta t^{\alpha}\right)\right] .
$$

Since $0 \leq E_{\alpha}\left(-\delta t^{\alpha}\right) \leq 1$, we have

$$
N(t) \leq N(0)+\frac{\lambda}{\delta} .
$$

The third equation of system (1) implies that

$$
\begin{aligned}
V(t)= & V(0) E_{\alpha}\left(-\mu t^{\alpha}\right) \\
& +k \int_{0}^{t} I(s) \alpha(t-s)^{\alpha-1} \frac{d E_{\alpha}}{d s}\left(-\mu(t-s)^{\alpha}\right) d s .
\end{aligned}
$$

Then,

$$
V(t) \leq V(0) E_{\alpha}\left(-\mu t^{\alpha}\right)+\frac{k\|I\|_{\infty}}{\mu}\left(1-E_{\alpha}\left(-\mu t^{\alpha}\right)\right) .
$$

Consequently,

$$
V(t) \leq V(0)+\frac{k\|I\|_{\infty}}{\mu} .
$$

This completes the proof.

\section{Equilibria and Their Local Stability}

It is easy to see that system (1) always has a disease-free equilibrium $E_{0}(\lambda / d, 0,0)$. Therefore, the basic reproduction number of our system (1) is given by

$$
R_{0}=\frac{k \beta \lambda}{\mu(a+\rho)\left(d+\lambda \alpha_{1}\right)} .
$$

Biologically, this basic reproduction number represents the average number of secondary infections produced by one infected cell during the period of infection when all cells are uninfected. Further, it is not hard to get the following result. 
Theorem 8. (i) If $R_{0} \leq 1$, system (1) has a unique diseasefree equilibrium of the form $E_{0}\left(T_{0}, 0,0\right)$, where $T_{0}=\lambda / d$. (ii) If $R_{0}>1$, the disease-free equilibrium is still present and system (1) has a unique chronic infection equilibrium of the form $E_{1}\left(T_{1},\left(\lambda-d T_{1}\right) / a, k\left(\lambda-d T_{1}\right) / a \mu\right)$, where $T_{1}=2(a+$ $\rho)\left(a \mu+\alpha_{2} \lambda k\right) /\left(a k \beta+(a+\rho)\left(\alpha_{2} d k-\alpha_{1} a \mu-\alpha_{3} k \lambda\right)+\sqrt{\bar{\delta}}\right)$ with

$$
\begin{aligned}
\bar{\delta}= & \left(a k \beta+(a+\rho)\left(\alpha_{2} d k-\alpha_{1} a \mu-\alpha_{3} k \lambda\right)\right)^{2} \\
& +4 \alpha_{3} k d(a+\rho)^{2}\left(a \mu+\alpha_{2} \lambda k\right) .
\end{aligned}
$$

Next, we investigate the local stability of equilibria. Let $E_{e}(T, I, V)$ be an arbitrary equilibrium of system (1). Then, the characteristic equation at $E_{e}$ is given by

$$
\begin{gathered}
\left|\begin{array}{ccc}
-d-V \frac{\partial f}{\partial T}-\xi & \rho & -V \frac{\partial f}{\partial V}-f(T, V) \\
V \frac{\partial f}{\partial T} & -(a+\rho)-\xi & V \frac{\partial f}{\partial V}+f(T, V) \\
0 & k & -\mu-\xi
\end{array}\right| \\
=0,
\end{gathered}
$$

where

$$
f(T, V)=\frac{\beta T}{1+\alpha_{1} T+\alpha_{2} V+\alpha_{3} T V} .
$$

We recall that the equilibrium $E_{e}$ is locally asymptotically stable if all roots $\xi_{i}$ of (31) satisfy the following condition [19]:

$$
\left|\arg \left(\xi_{i}\right)\right|>\frac{\alpha \pi}{2}
$$

Theorem 9. (i) If $R_{0}<1$, then $E_{0}$ is locally asymptotically stable. (ii) If $R_{0}>1$, then $E_{0}$ is unstable.

Proof. Evaluating (31) at $E_{0}$, we have

$$
(d+\xi)\left[\xi^{2}+(a+\rho+\mu) \xi+\mu(a+\rho)\left(1-R_{0}\right)\right]=0
$$

Obviously, the roots of (34) are

$$
\begin{aligned}
& \xi_{1}=-d, \\
& \xi_{2} \\
& =\frac{-(a+\rho+\mu)-\sqrt{(a+\rho+\mu)^{2}-4 \mu(a+\rho)\left(1-R_{0}\right)}}{2}, \\
& \xi_{3} \\
& =\frac{-(a+\rho+\mu)+\sqrt{(a+\rho+\mu)^{2}-4 u(a+\rho)\left(1-R_{0}\right)}}{2} .
\end{aligned}
$$

It is clear that $\xi_{1}$ and $\xi_{2}$ are negative. However, $\xi_{3}$ is negative if $R_{0}<1$ and it is positive if $R_{0}>1$. Therefore, $E_{0}$ is locally asymptotically stable if $R_{0}<1$ and unstable if $R_{0}>1$.
Now, we focus on the local stability of the chronic infection equilibrium $E_{1}$. It follows from (31) that the characteristic equation at $E_{1}$ is given by

$$
P(\xi):=\xi^{3}+a_{1} \xi^{2}+a_{2} \xi+a_{3}=0,
$$

where

$$
\begin{aligned}
a_{1}= & \mu+d+a+\rho+\frac{\beta V_{1}\left(1+\alpha_{2} V_{1}\right)}{\left(1+\alpha_{1} T_{1}+\alpha_{2} V_{1}+\alpha_{3} T_{1} V_{1}\right)^{2}}, \\
a_{2}= & d(\mu+a+\rho) \\
& +\frac{\beta V_{1}\left[(a+\mu)\left(1+\alpha_{2} V_{1}\right)+k T_{1}\left(\alpha_{2}+\alpha_{3} T_{1}\right)\right]}{\left(1+\alpha_{1} T_{1}+\alpha_{2} V_{1}+\alpha_{3} T_{1} V_{1}\right)^{2}}, \\
a_{3}= & \frac{\beta V_{1}\left[a \mu\left(1+\alpha_{2} V_{1}\right)+k d T_{1}\left(\alpha_{2}+\alpha_{3} T_{1}\right)\right]}{\left(1+\alpha_{1} T_{1}+\alpha_{2} V_{1}+\alpha_{3} T_{1} V_{1}\right)^{2}} .
\end{aligned}
$$

It is obvious that $a_{1}>0, a_{2}>0$, and $a_{3}>0$. Further, we have

$$
\begin{aligned}
& a_{1} a_{2}-a_{3}=a(d(\mu+a+\rho) \\
& \left.+\frac{\beta V_{1}\left[a\left(1+\alpha_{2} V_{1}\right)+k T_{1}\left(\alpha_{2}+\alpha_{3} T_{1}\right)\right]}{\left(1+\alpha_{1} T_{1}+\alpha_{2} V_{1}+\alpha_{3} T_{1} V_{1}\right)^{2}}\right) \\
& +d(d(\mu+a+\rho) \\
& \left.+\frac{(a+\mu) \beta V_{1}\left(1+\alpha_{2} V_{1}\right)}{\left(1+\alpha_{1} T_{1}+\alpha_{2} V_{1}+\alpha_{3} T_{1} V_{1}\right)^{2}}\right)+(\mu+\rho \\
& \left.+\frac{\beta V_{1}\left(1+\alpha_{2} V_{1}\right)}{\left(1+\alpha_{1} T_{1}+\alpha_{2} V_{1}+\alpha_{3} T_{1} V_{1}\right)^{2}}\right) h_{2}>0 .
\end{aligned}
$$

So, Routh-Hurwitz conditions are satisfied. Let $D(P)$ denote the discriminant of the polynomial $P$ given by (36); then,

$$
D(P)=18 a_{1} a_{2} a_{3}+\left(a_{1} a_{2}\right)^{2}-4 a_{3} a_{1}^{3}-4 a_{2}^{3}-27 a_{3}^{2} .
$$

Using the results in [19], we easily obtain the following result.

Theorem 10. Assume that $R_{0}>1$.

(i) If $D(P)>0$, then $E_{1}$ is locally asymptotically stable for all $\alpha \in(0,1]$.

(ii) If $D(P)<0$ and $\alpha<2 / 3$, then $E_{1}$ is locally asymptotically stable.

\section{Global Stability}

In this section, we study the global stability of the disease-free equilibrium $E_{0}$ and the chronic infection equilibrium $E_{1}$.

Theorem 11. If $R_{0} \leq 1$, then the disease-free equilibrium $E_{0}$ is globally asymptotically stable. 
Proof. Define Lyapunov functional $L_{0}(t)$ as follows:

$$
\begin{aligned}
L_{0}(t)= & \frac{T_{0}}{1+\alpha_{1} T_{0}} \Phi\left(\frac{T}{T_{0}}\right) \\
& +\frac{\rho}{2\left(1+\alpha_{1} T_{0}\right)(a+d) T_{0}}\left(T-T_{0}+I\right)^{2} \\
& +\frac{a+\rho}{k} V,
\end{aligned}
$$

where $\Phi(x)=x-1-\ln (x), x>0$. Calculating the derivative of $L_{0}(t)$ along solutions of system (1) and using the results in [20], we get

$$
\begin{aligned}
D^{\alpha} L_{0}(t) \leq & \frac{1}{1+\alpha_{1} T_{0}}\left(1-\frac{T_{0}}{T}\right) D^{\alpha} T+D^{\alpha} I \\
& \cdot \frac{\rho}{\left(1+\alpha_{1} T_{0}\right)(a+d) T_{0}}\left(T-T_{0}+I\right) \\
& \cdot\left(D^{\alpha} T+D^{\alpha} I\right)+\frac{a+\rho}{k} D^{\alpha} V
\end{aligned}
$$

Using $\lambda=d T_{0}$, we obtain

$$
\begin{aligned}
D^{\alpha} L_{0}(t) & \\
\leq & -\frac{d\left(T-T_{0}\right)^{2}}{\left(1+\alpha_{1} T_{0}\right) T}-\frac{1}{1+\alpha_{1} T_{0}}\left(1-\frac{T_{0}}{T}\right) f(T, V) V \\
& +\frac{\rho}{1+\alpha_{1} T_{0}}\left(1-\frac{T_{0}}{T}\right) I+f(T, V) V \\
& -\frac{d \rho\left(T-T_{0}\right)^{2}}{(a+d) T_{0}\left(1+\alpha_{1} T_{0}\right)} \\
& -\frac{a \rho I^{2}}{(a+d) T_{0}\left(1+\alpha_{1} T_{0}\right)} \\
& +\frac{\rho}{T_{0}\left(1+\alpha_{1} T_{0}\right)} I\left(T_{0}-T\right)-\frac{(a+\rho) \mu}{k} V
\end{aligned}
$$

$$
\begin{aligned}
D^{\alpha} L_{0}(t) & \\
\leq & -\left(\frac{1}{T}+\frac{\rho}{(a+d) T_{0}}\right) \frac{d\left(T-T_{0}\right)^{2}}{1+\alpha_{1} T_{0}} \\
& -\frac{\rho I\left(T-T_{0}\right)^{2}}{T T_{0}\left(1+\alpha_{1} T_{0}\right)}-\frac{a \rho I^{2}}{(a+d) T_{0}\left(1+\alpha_{1} T_{0}\right)} \\
& +\frac{(a+\rho) \mu}{k}\left(R_{0}-1\right) V \\
& -\frac{\beta T_{0}\left(\alpha_{2}+\alpha_{3} T\right)}{\left(1+\alpha_{1} T_{0}\right)\left(1+\alpha_{1} T+\alpha_{2} V+\alpha_{3} T V\right)} V^{2} .
\end{aligned}
$$

Hence, if $R_{0} \leq 1$, then $D^{\alpha} L_{0}(t) \leq 0$. Furthermore, it is clear that the largest invariant set of $\left\{(T, I, V) \in D: D^{\alpha} L_{0}(t)=0\right\}$ is the singleton $\left\{E_{0}\right\}$. Therefore, by LaSalle's invariance principle [21], $E_{0}$ is globally asymptotically stable.
Theorem 12. The chronic infection equilibrium $E_{1}$ is globally asymptotically stable if $R_{0}>1$ and

$$
R_{0} \leq 1+\frac{d(a+\rho)\left(\lambda \alpha_{2} k+a \mu\right)+\rho \lambda^{2} \alpha_{3} k}{\rho \mu(a+\rho)\left(d+\lambda \alpha_{1}\right)} .
$$

Proof. Define Lyapunov functional $L_{1}(t)$ as follows:

$$
\begin{aligned}
& L_{1}(t)=\frac{1+\alpha_{2} V_{1}}{1+\alpha_{1} T_{1}+\alpha_{2} V_{1}+\alpha_{3} T_{1} V_{1}} T_{1} \Phi\left(\frac{T}{T_{1}}\right) \\
& +I_{1} \Phi\left(\frac{I}{I_{1}}\right)+\frac{a+\rho}{k} V_{1} \Phi\left(\frac{V}{V_{1}}\right) \\
& +\frac{\rho\left(1+\alpha_{2} V_{1}\right)}{2 T_{1}(a+d)\left(1+\alpha_{1} T_{1}+\alpha_{2} V_{1}+\alpha_{3} T_{1} V_{1}\right)}(T \\
& \left.-T_{1}+I-I_{1}\right)^{2} .
\end{aligned}
$$

Then, we have

$$
\begin{aligned}
& D^{\alpha} L_{1}(t) \leq \frac{1+\alpha_{2} V_{1}}{1+\alpha_{1} T_{1}+\alpha_{2} V_{1}+\alpha_{3} T_{1} V_{1}}\left(1-\frac{T_{1}}{T}\right) \\
& \cdot D^{\alpha} T+\left(1-\frac{I_{1}}{I}\right) D^{\alpha} I \\
& \cdot \frac{\rho\left(1+\alpha_{2} V_{1}\right)}{T_{1}(a+d)\left(1+\alpha_{1} T_{1}+\alpha_{2} V_{1}+\alpha_{3} T_{1} V_{1}\right)}\left(T-T_{1}\right. \\
& \left.+I-I_{1}\right)\left(D^{\alpha} T+D^{\alpha} I\right)+\frac{a+\rho}{k}\left(1-\frac{V_{1}}{V}\right) D^{\alpha} V .
\end{aligned}
$$

Using $\lambda=d T_{1}+a I_{1}, f\left(T_{1}, V_{1}\right) V_{1}=(a+\rho) I_{1}, \mu / k=I_{1} / V_{1}$, and $1-f\left(T_{1}, V_{1}\right) / f\left(T, V_{1}\right)=\left(\left(1+\alpha_{2} V_{1}\right) /\left(1+\alpha_{1} T_{1}+\alpha_{2} V_{1}+\right.\right.$ $\left.\left.\alpha_{3} T_{1} V_{1}\right)\right)\left(1-T_{1} / T\right)$, we get

$$
\begin{aligned}
& D^{\alpha} L_{1}(t) \leq d\left(1-\frac{f\left(T_{1}, V_{1}\right)}{f\left(T, V_{1}\right)}\right)\left(T_{1}-T\right)+(a+\rho) \\
& \cdot I_{1}\left(4-\frac{f\left(T_{1}, V_{1}\right)}{f\left(T, V_{1}\right)}-\frac{I_{1}}{I} \frac{V}{V_{1}} \frac{f(T, V)}{f\left(T_{1}, V_{1}\right)}-\frac{I}{I_{1}} \frac{V_{1}}{V}\right. \\
& \left.-\frac{f\left(T, V_{1}\right)}{f(T, V)}\right)+(a+\rho) I_{1}\left(-1-\frac{V}{V_{1}}+\frac{f\left(T, V_{1}\right)}{f(T, V)}\right. \\
& \left.+\frac{V}{V_{1}} \frac{f(T, V)}{f\left(T, V_{1}\right)}\right) \\
& -\frac{d \rho\left(1+\alpha_{2} V_{1}\right)}{T_{1}(a+d)\left(1+\alpha_{1} T_{1}+\alpha_{2} V_{1}+\alpha_{3} T_{1} V_{1}\right)}(T \\
& \left.-T_{1}\right)^{2} \\
& -\frac{a \rho\left(1+\alpha_{2} V_{1}\right)}{T_{1}(a+d)\left(1+\alpha_{1} T_{1}+\alpha_{2} V_{1}+\alpha_{3} T_{1} V_{1}\right)}\left(I-I_{1}\right)^{2} \\
& -\frac{\rho\left(1+\alpha_{2} V_{1}\right)}{T T_{1}\left(1+\alpha_{1} T_{1}+\alpha_{2} V_{1}+\alpha_{3} T_{1} V_{1}\right)}\left(T-T_{1}\right)^{2}(I \\
& \left.-I_{1}\right) \text {. }
\end{aligned}
$$



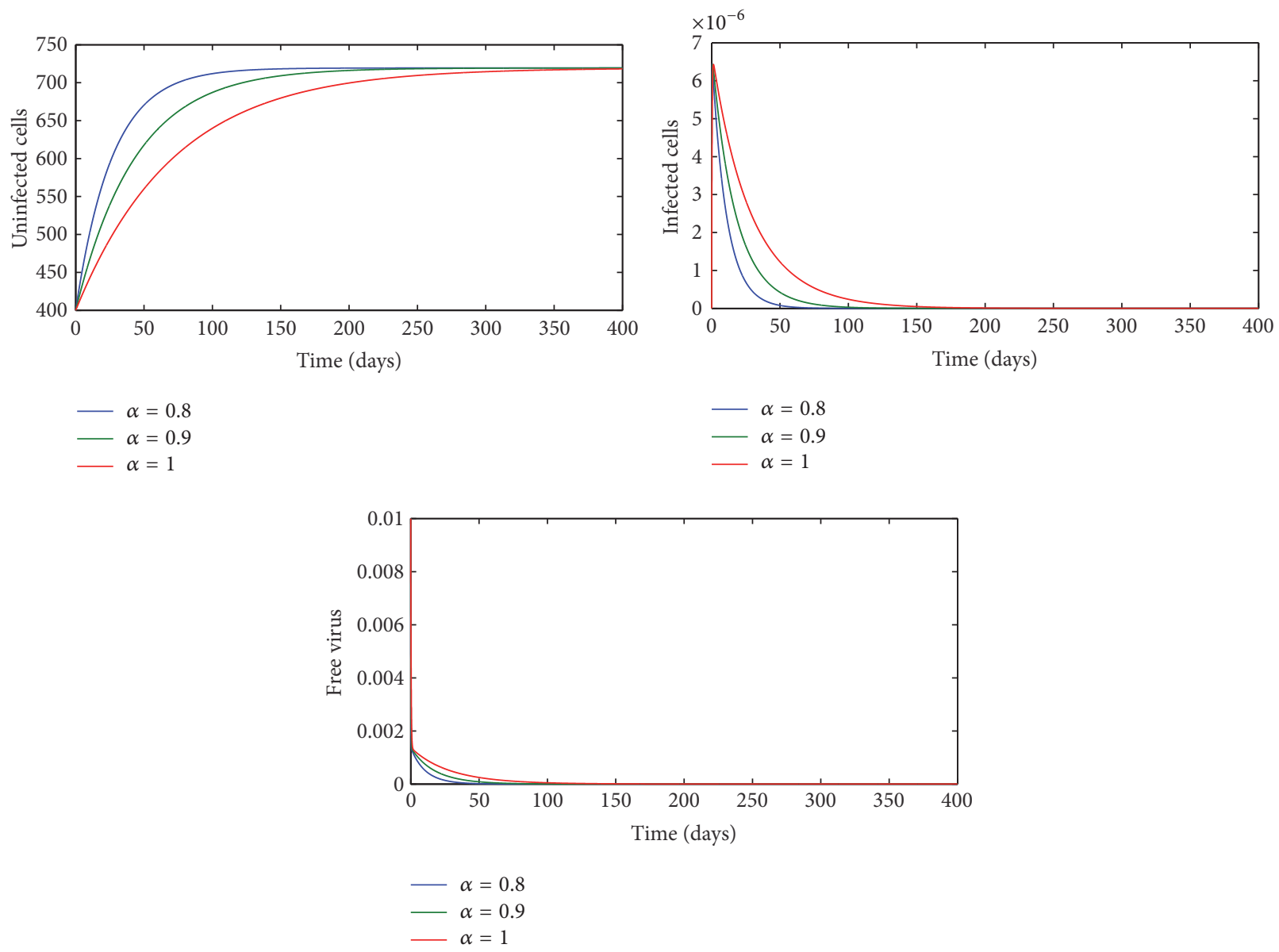

FIGURE 1: Stability of the disease-free equilibrium $E_{0}$.

Thus,

$$
\begin{aligned}
& D^{\alpha} L_{1}(t) \\
& \quad \leq-\frac{\left(1+\alpha_{2} V_{1}\right)\left(T-T_{1}\right)^{2}}{T T_{1}\left(1+\alpha_{1} T_{1}+\alpha_{2} V_{1}+\alpha_{3} T_{1} V_{1}\right)}\left(\left(d T_{1}-\rho I_{1}\right)\right. \\
& \left.\quad+\frac{d \rho T}{d+a}+\rho I\right)-(a+\rho) I_{1} \\
& \cdot \frac{\left(1+\alpha_{1} T\right)\left(\alpha_{2}+\alpha_{3} T\right)\left(V-V_{1}\right)^{2}}{V_{1}\left(1+\alpha_{1} T+\alpha_{2} V+\alpha_{3} T V\right)\left(1+\alpha_{1} T+\alpha_{2} V_{1}+\alpha_{3} T V_{1}\right)} \\
& -(a+\rho) I_{1}\left(\Phi\left(\frac{f\left(T_{1}, V_{1}\right)}{f\left(T, V_{1}\right)}\right)+\Phi\left(\frac{I_{1}}{I} \frac{V}{V_{1}} \frac{f(T, V)}{f\left(T_{1}, V_{1}\right)}\right)\right. \\
& \left.+\Phi\left(\frac{I}{I_{1}} \frac{V_{1}}{V}\right)+\Phi\left(\frac{f\left(T, V_{1}\right)}{f(T, V)}\right)\right) \\
& -\frac{a \rho\left(1+\alpha_{2} V_{1}\right)}{T_{1}(a+d)\left(1+\alpha_{1} T_{1}+\alpha_{2} V_{1}+\alpha_{3} T_{1} V_{1}\right)}\left(I-I_{1}\right)^{2} .
\end{aligned}
$$

It is clear that $\Phi(x) \geq 0$. Consequently, $D^{\alpha} L_{1}(t) \leq 0$ if $d T_{1} \geq \rho I_{1}$. In addition, it is easy to see that this condition is equivalent to (43). Further, the largest invariant set of $\left\{(T, I, V) \in D: D^{\alpha} L_{1}(t)=0\right\}$ is the singleton $\left\{E_{1}\right\}$. By
LaSalle's invariance principle, $E_{1}$ is globally asymptotically stable.

It is important to see that

$$
\lim _{\rho \rightarrow 0} \frac{d(a+\rho)\left(\lambda \alpha_{2} k+a \mu\right)+\rho \lambda^{2} \alpha_{3} k}{\rho \mu(a+\rho)\left(d+\lambda \alpha_{1}\right)}=+\infty
$$

According to Theorem 12, we obtain the following result.

Corollary 13. The chronic infection equilibrium $E_{1}$ is globally asymptotically stable when $R_{0}>1$ and $\rho$ is sufficiently small.

\section{Numerical Simulations}

In this section, we give some numerical simulations in order to illustrate our theoretical results. We discretize system (1) by using fractional Euler's method presented in [22]. Firstly, we take the parameter values as shown in Table 1.

By calculation, we have $R_{0}=0.9283<1$. Then, system (1) has a disease-free equilibrium $E_{0}(719.4245,0,0)$. By Theorem 11, the solution of (1) converges to $E_{0}$ (see Figure 1). Consequently, the virus is cleared and the infection dies out. 

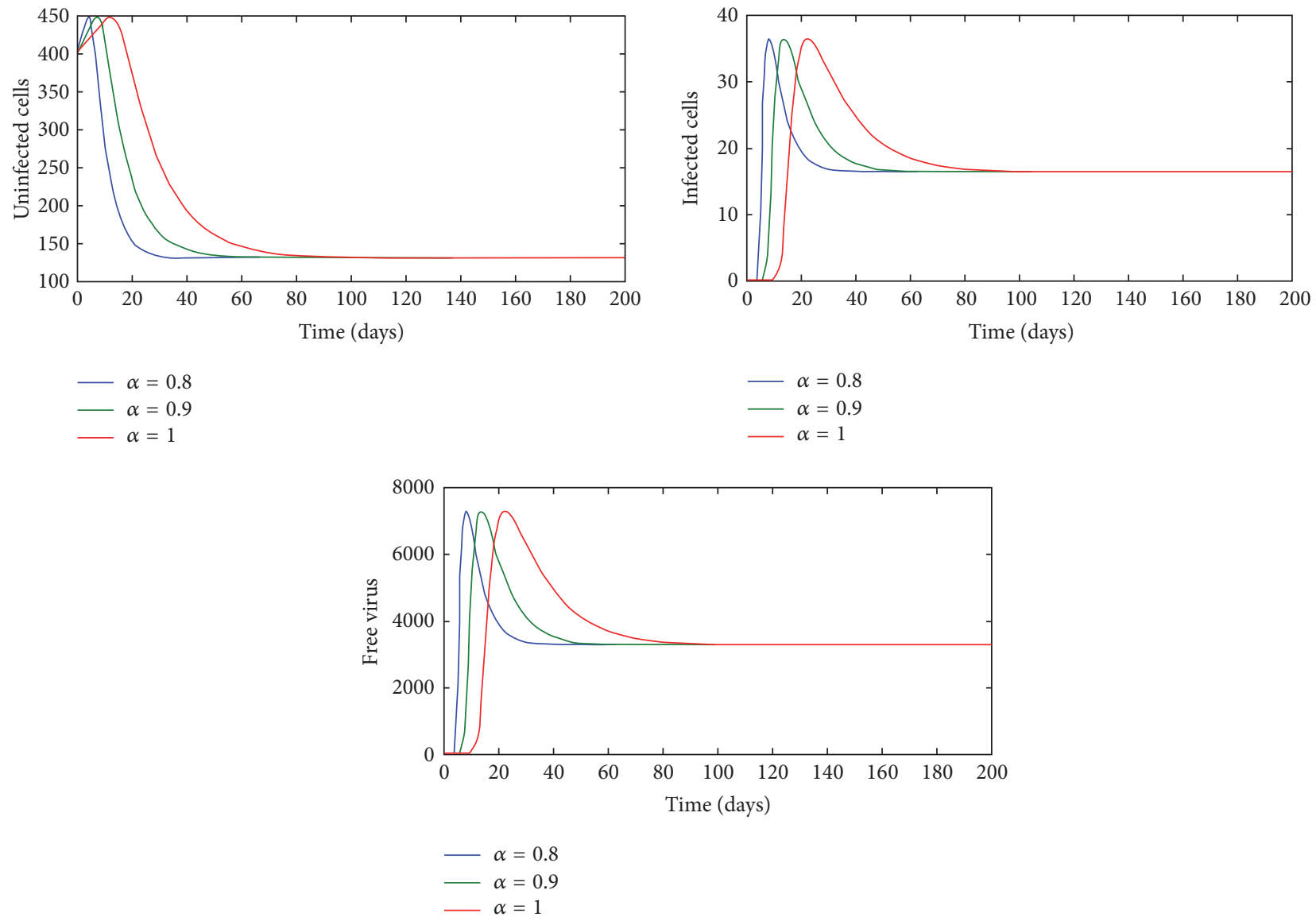

FIgURE 2: Stability of the chronic infection equilibrium $E_{1}$.

TABLE 1: Parameter values of system (1).

\begin{tabular}{lc}
\hline Parameters & Values \\
\hline$\lambda$ & 10 \\
$d$ & 0.0139 \\
$\beta$ & 0.00024 \\
$\rho$ & 0.01 \\
$a$ & 0.5 \\
$k$ & 600 \\
$u$ & 3 \\
$\alpha_{1}$ & 0.1 \\
$\alpha_{2}$ & 0.01 \\
$\alpha_{3}$ & 0.00001 \\
\hline
\end{tabular}

Now, we choose $\beta=0.001$ and we keep the other parameter values. In this case, $R_{0}=3.8678$ and

$$
1+\frac{d(a+\rho)\left(\lambda \alpha_{2} k+a \mu\right)+\rho \lambda^{2} \alpha_{3} k}{\rho \mu(a+\rho)\left(d+\lambda \alpha_{1}\right)}=415.885
$$

Hence, condition (43) is satisfied. Therefore, the chronic infection equilibrium $E_{1}(130.1613,16.3815,3276.3)$ is globally asymptotically stable. Figure 2 demonstrates this result.

\section{Conclusion}

In this paper, we have proposed a fractional order model of HIV infection with specific functional response and cure rate. This functional response covers the most functional responses used by several authors such as the saturated incidence rate, the Beddington-DeAngelis functional response, and the Crowley-Martin functional response. We have shown that the proposed model has a bounded and nonnegative solution as desired in any population dynamics. By using stability analysis of fractional order system, we have proved that if the basic reproduction number $R_{0} \leq 1$, the diseasefree equilibrium $E_{0}$ is globally asymptotically stable for all $\alpha \epsilon$ $(0,1]$, which means that the virus is cleared and the infection dies out. However, when $R_{0}>1$, the disease-free equilibrium $E_{0}$ becomes unstable and there exists another biological equilibrium, namely, chronic infection equilibrium $E_{1}$, that is globally asymptotically stable provided that condition (43) is satisfied. In this case, the HIV virus persists in the host and the infection becomes chronic. Furthermore, we have remarked that if the cure rate $\rho$ is equal to zero or is sufficiently small, condition (43) is satisfied and the global stability of $E_{1}$ is only characterized by $R_{0}>1$.

According to the above theoretical analysis, we deduce that the global dynamics of the model are fully determined by the basic reproduction number $R_{0}$. In addition, we see 
that the fractional order parameter $\alpha$ has no effect on the global dynamics of our model, but it can affect the time for arriving at both steady states (see Figures 1 and 2). Moreover, the fractional order model and main results presented by Liu et al. in [11] are generalized and improved.

\section{Conflicts of Interest}

The authors declare that there are no conflicts of interest regarding the publication of this paper.

\section{References}

[1] Y. A. Rossikhin and M. V. Shitikova, "Applications of fractional calculus to dynamic problems of linear and nonlinear hereditary mechanics of solids," Applied Mechanics Reviews, vol. 50, no. 1, pp. 15-67, 1997.

[2] R. J. Marks and M. W. Hall, "Differintegral Interpolation from a Bandlimited Signal's Samples," IEEE Transactions on Acoustics, Speech, and Signal Processing, vol. 29, no. 4, pp. 872-877, 1981.

[3] G. L. Jia and Y. X. Ming, "Study on the viscoelasticity of cancellous bone based on higher-order fractional models," in Proceedings of the 2nd International Conference on Bioinformatics and Biomedical Engineering, iCBBE 2008, pp. 1733-1736, chn, May 2008.

[4] R. Magin, Fractional Calculus in Bioengineering, Cretical Reviews in Biomedical Engineering 32, vol. 32, 2004.

[5] E. Scalas, R. Gorenflo, and F. Mainardi, "Fractional calculus and continuous-time finance," Physica A. Statistical Mechanics and its Applications, vol. 284, no. 1-4, pp. 376-384, 2000.

[6] L. Song, S. Xu, and J. Yang, "Dynamical models of happiness with fractional order," Communications in Nonlinear Science and Numerical Simulation, vol. 15, no. 3, pp. 616-628, 2010.

[7] R. Capponetto, G. Dongola, L. Fortuna, and I. Petras, "Fractional order systems: Modelling and control applications," in World Scientific Series in Nonlinear Science, vol. 72, Series A, Singapore, 2010.

[8] K. S. Cole, "Electric conductance of biological systems," Cold Spring Harbor Symposia on Quantitative Biology, vol. 1, pp. 107116, 1933.

[9] A. A. Arafa, S. Z. Rida, and M. Khalil, "A fractional-order model of HIV infection: numerical solution and comparisons with data of patients," International Journal of Biomathematics, vol. 7, no. 4, Article ID 1450036, 1450036, 11 pages, 2014.

[10] A. A. M. Arafa, S. Z. Rida, and M. Khalil, "Fractional modeling dynamics of HIV and CD4 + T-cells during primary infection," Nonlinear Biomedical Physics, vol. 6, no. 1, article 1, 2012.

[11] Y. Liu, J. Xiong, C. Hu, and C. Wu, "Stability analysis for fractional differential equations of an HIV infection model with cure rate," in Proceedings of the 2016 IEEE International Conference on Information and Automation, IEEE ICIA 2016, pp. 707-711, August 2016.

[12] X. Zhou and J. Cui, "Global stability of the viral dynamics with Crowley-Martin functional response," Bulletin of the Korean Mathematical Society, vol. 48, no. 3, pp. 555-574, 2011.

[13] G. Huang, W. Ma, and Y. Takeuchi, "Global properties for virus dynamics model with Beddington-DeAngelis functional response," Applied Mathematics Letters. An International Journal of Rapid Publication, vol. 22, no. 11, pp. 1690-1693, 2009.
[14] M. A. Nowak and C. R. M. Bangham, "Population dynamics of immune responses to persistent viruses," Science, vol. 272, no. 5258, pp. 74-79, 1996.

[15] P. K. Srivastava and P. Chandra, "Modeling the dynamics of HIV and $\mathrm{CD} 4{ }^{+} \mathrm{T}$ cells during primary infection," Nonlinear Analysis. Real World Applications. An International Multidisciplinary Journal, vol. 11, no. 2, pp. 612-618, 2010.

[16] I. Podlubny, Fractional Differential Equations, vol. 198 of Mathematics in Science and Engineering, Academic Press, San Diego, Calif, USA, 1999.

[17] W. Lin, "Global existence theory and chaos control of fractional differential equations," Journal of Mathematical Analysis and Applications, vol. 332, no. 1, pp. 709-726, 2007.

[18] Z. M. Odibat and N. T. Shawagfeh, "Generalized Taylor's formula," Applied Mathematics and Computation, vol. 186, no. 1, pp. 286-293, 2007.

[19] E. Ahmed, A. M. El-Sayed, and H. A. El-Saka, "On some RouthHurwitz conditions for fractional order differential equations and their applications in Lorenz, Rossler, Chua and CHEn systems," Physics Letters. A, vol. 358, no. 1, pp. 1-4, 2006.

[20] C. V. De-Leon, "Volterra-type Lyapunov functions for fractional-order epidemic systems," Communications in Nonlinear Science and Numerical Simulation, vol. 24, no. 1-3, pp. 75-85, 2015.

[21] J. Huo, H. Zhao, and L. Zhu, "The effect of vaccines on backward bifurcation in a fractional order HIV model," Nonlinear Analysis. Real World Applications, vol. 26, pp. 289-305, 2015.

[22] Z. Odibat and S. Momani, "An algorithm for the numerical solution of differential equations of fractional order," Applied Mathematics \& Information, vol. 26, no. 1, pp. 15-27, 2008. 


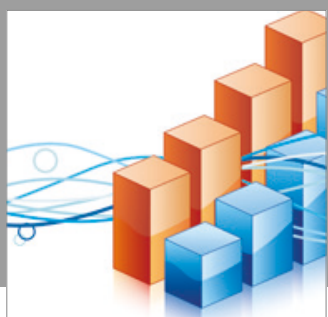

Advances in

Operations Research

vatersals

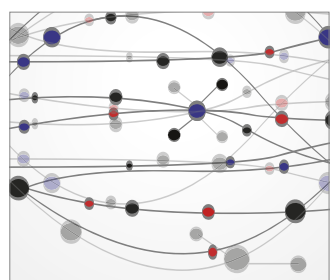

\section{The Scientific} World Journal
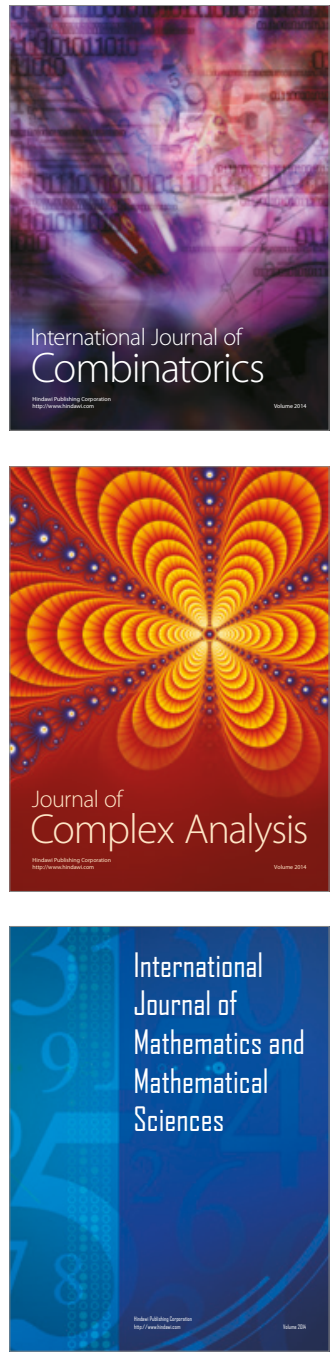
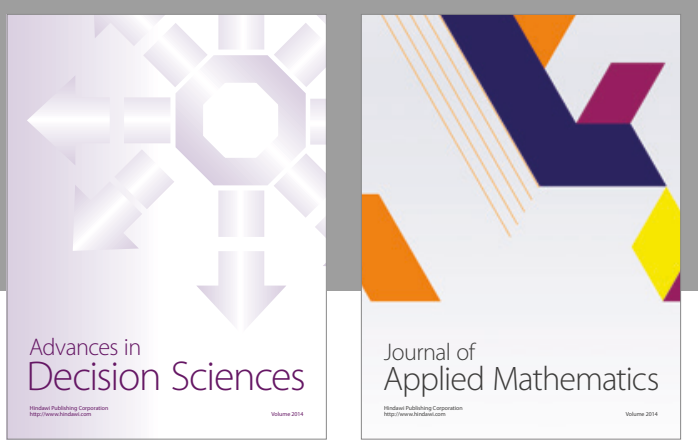

Algebra

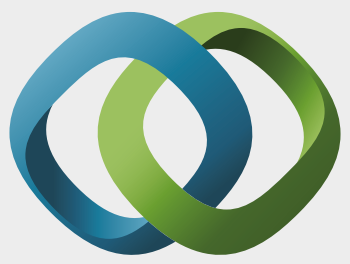

\section{Hindawi}

Submit your manuscripts at

https://www.hindawi.com
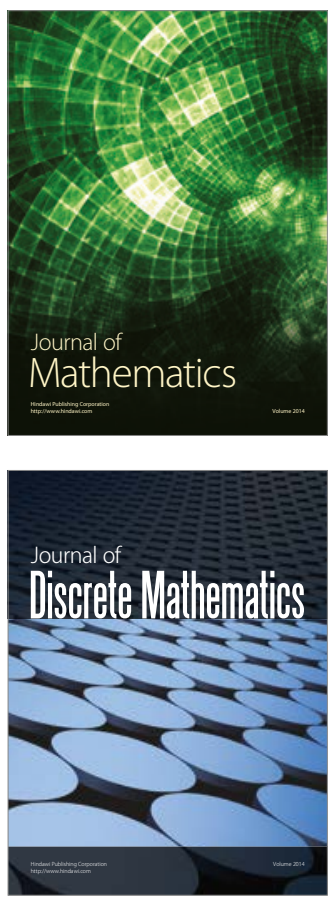

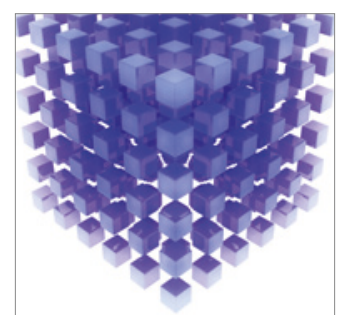

Mathematical Problems in Engineering
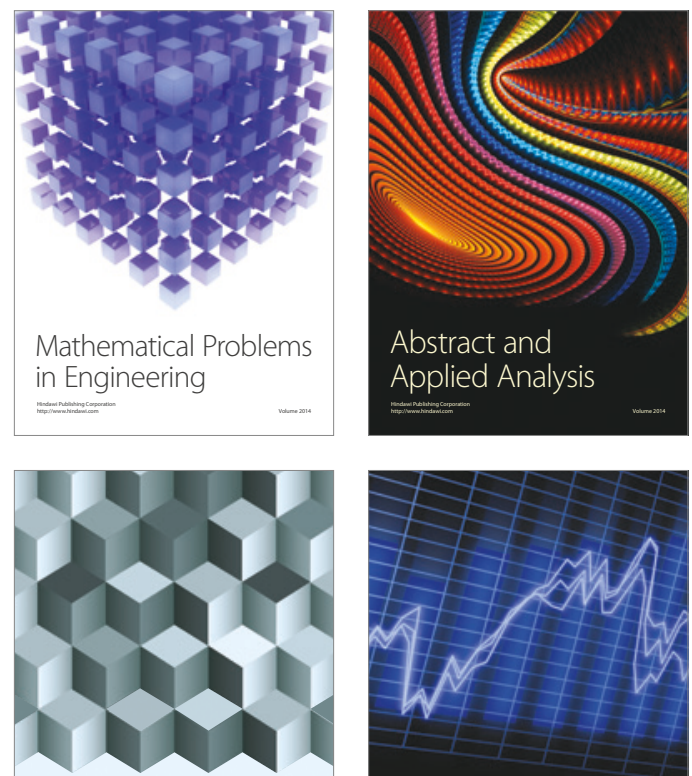

Journal of

Function Spaces

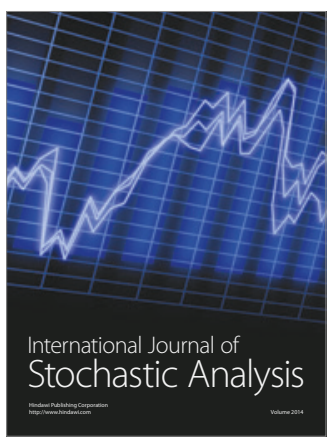

Probability and Statistics
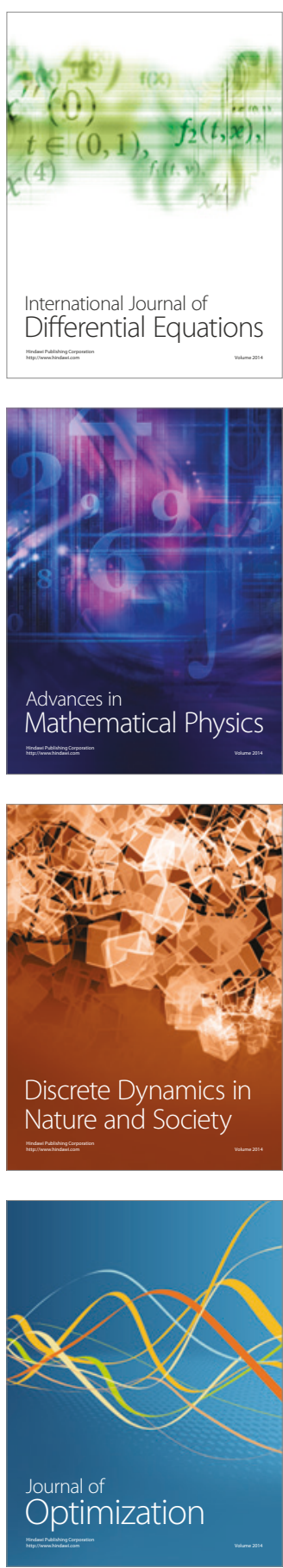